\title{
Infinite hierarchy of solitons: Interaction of Kerr nonlinearity with even orders of dispersion
}

\author{
Antoine F. J. Runge $\odot,{ }^{1,}{ }^{*}$ Y. Long Qiang $\odot,{ }^{1}$ Tristram J. Alexander, ${ }^{1}$ M. Z. Rafat $\odot,{ }^{1}$ Darren D. Hudson, ${ }^{2}$ \\ Andrea Blanco-Redondo, ${ }^{3}$ and C. Martijn de Sterke ${ }^{1,4}$ \\ ${ }^{1}$ Institute of Photonics and Optical Science (IPOS), School of Physics, University of Sydney, NSW 2006, Australia \\ ${ }^{2}$ CACI-Photonics Solutions, 15 Vreeland Road, Florham Park, New Jersey 07932, USA \\ ${ }^{3}$ Nokia Bell Labs, 791 Holmdel Road, Holmdel, New Jersey 07733, USA \\ ${ }^{4}$ University of Sydney Nano Institute (Sydney Nano), University of Sydney, NSW 2006, Australia
}

(Received 11 December 2020; accepted 5 February 2021; published 22 February 2021)

\begin{abstract}
Temporal solitons are optical pulses that arise from the balance of negative group-velocity dispersion and self-phase modulation. For decades, only quadratic dispersion was considered with higher order dispersion often thought of as a nuisance. Following the recent observation of pure-quartic solitons, we here provide experimental and numerical evidence for an infinite hierarchy of solitons that balance self-phase modulation and arbitrary negative pure, even-order dispersion. Specifically, we experimentally demonstrate the existence of solitons with pure-sextic $\left(\beta_{6}\right)$, -octic $\left(\beta_{8}\right)$, and -decic $\left(\beta_{10}\right)$ dispersion, limited only by the performance of our components, and we numerically show the existence of solitons involving pure 16th-order dispersion. These results broaden the fundamental understanding of solitons and present avenues to engineer ultrafast pulses in nonlinear optics and its applications.
\end{abstract}

DOI: 10.1103/PhysRevResearch.3.013166

\section{INTRODUCTION}

Solitons are among the most striking phenomena in nonlinear physics and have been observed in a wide range of systems $[1,2]$. In optics, these transform-limited, shape-maintaining pulses have been crucial in the development of numerous applications ranging from telecommunications $[3,4]$, to frequency comb generation [5,6] and mode-locked lasers [7,8]. Traditionally, the formation and propagation of these wave packets rely on the balance between self-phase modulation (SPM) and negative quadratic dispersion $\left(\beta_{2}<0\right)$, while higher dispersion orders were seen as a nuisance, leading to dispersive wave emission in fibers $[9,10]$ and laser cavities $[11,12]$, or acting to limit the achievable pulse duration [13-16].

When a pulse propagates through a positive nonlinear medium, SPM causes the generation of new low frequencies on the leading edge of the pulse and new high frequencies on its trailing edge. To understand solitons, recall that in the presence of pure negative dispersion $\beta_{k}$ of order $k$ at frequency $\omega_{0}$, the inverse group velocity $v_{g}$ for frequencies close to $\omega_{0}$ can be written as

$$
\frac{1}{v_{g}}=\frac{1}{v_{g 0}}-\frac{1}{(k-1) !}\left|\beta_{k}\right|\left(\omega-\omega_{0}\right)^{k-1} .
$$

\footnotetext{
*Corresponding author: antoine.runge@ sydney.edu.au

Published by the American Physical Society under the terms of the Creative Commons Attribution 4.0 International license. Further distribution of this work must maintain attribution to the author(s) and the published article's title, journal citation, and DOI.
}

Here $v_{g 0}$ is the group velocity at $\omega_{0}$, and $k=2$ for quadratic dispersion, etc. For negative quadratic and in fact for all higher, even-order types of negative dispersion, Eq. (1) shows that the group velocity monotonically increases with frequency. Consequently, both the SPM-generated low frequencies on the leading edge and the SPM-generated high frequencies on the trailing edge move towards the pulse center, leading to the formation of a soliton. This argument suggests that temporal solitons should exist in the presence of any negative even-order dispersion. Indeed, recent studies showed that optical solitons could arise from the balance between SPM and negative quartic dispersion $\left(\beta_{4}<0\right)$ [17-19]. However, the existence of solitons for higher, even dispersion order $(k>4)$ and their properties are yet to be reported.

Here we report evidence for an infinite hierarchy of solitons of which conventional solitons and pure-quartic solitons are the two lowest-order members. Since they arise from the balance between SPM and a single negative even order of dispersion, we refer to them as pure high, even-order dispersion (PHEOD) solitons. This is to distinguish them from previous theoretical studies which considered the combined effects of high-order dispersion of order up to $k=8$ and nonlinear effects [20-28]. These studies all consider equations with a large number of terms, many of which represent combined nonlinear and dispersive effects. Our aim differs in that we consider the Kerr nonlinear effect and pure, high, even-order dispersion [see Eq. (2) below]. We found numerical solutions by solving the nonlinear Schrödinger equation (NLSE), modified to higher orders of dispersion. In this way we provide evidence of PHEOD solitons up to order 16, and we find that each order has a unique set of properties.

We then experimentally demonstrate three members of this hierarchy, namely, pure-sextic, -octic, and -decic solitons 
(arising, respectively, from the balance of SPM and negative dipsersion of order $k=6,8$, and 10), using a passively mode-locked laser with tunable net-cavity dispersion [19]. The experimental results agree well with numerical predictions. The solitons have spectral sidebands, associated with resonant dispersive waves typical of fiber lasers, the spacing of which is directly, and quantitatively, related to the dispersion. By measuring the PHEOD solitons' energy we find that they are related to the pulse duration, $\tau$, as $E \propto \tau^{-(k-1)}$. Our results establish a new degree of freedom for the generation and study of ultrashort optical pulses with potential applications in lasers [19] and frequency comb generation [29,30].

\section{NUMERICAL RESULTS}

\section{A. Numerical solutions}

We consider the propagation of optical pulses in a medium with Kerr nonlinearity and $k$ th-order dispersion, where $k$ is an even integer. This evolution can be described by the modified NLSE

$$
i \frac{\partial \psi}{\partial z}=-(i)^{k} \frac{\left|\beta_{k}\right|}{k !} \frac{\partial^{k} \psi}{\partial T^{k}}-\gamma|\psi|^{2} \psi,
$$

where $\psi(z, T)$ is the pulse envelope, $z$ is the propagation coordinate, $T$ is the local time, $\beta_{k}$ is the dispersion coefficient, which is taken to be negative, and $\gamma$ is the nonlinear parameter. For $k>2$, Eq. (2) has no known analytic pulselike solutions. However, we can look for stationary solutions of Eq. (2) of the form $\psi(z, T)=A_{k}(T ; \mu) e^{i \mu z}$, which satisfy

$$
\mu A_{k}-(i)^{k} \frac{\left|\beta_{k}\right|}{k !} \frac{\partial^{k} A_{k}}{\partial T^{k}}-\gamma A_{k}^{3}=0,
$$

so the shape is preserved during propagation, and $A_{k}$ can be taken to be real. We solve Eq. (3) using the Newton-conjugategradient method [18,31].

Solving Eq. (3) provides a single solution for each dispersion order, but using a scaling argument we can obtain an entire family of solutions. Since Eq. (2) is invariant under the transformation

$$
A_{k} \rightarrow \alpha A_{k}, \quad \tau \rightarrow \alpha^{-2 / k} \tau, \quad \mu \rightarrow \alpha^{2} \mu,
$$

there exists a continuous family of PHEOD solitons for each dispersion order $k$, each with the same pulse shape, but with different amplitudes and widths, that can be parameterized by the value of the nonlinear phase shift $\mu$ [18].

The numerically calculated temporal and spectral intensity profiles of the stationary solutions for $k=6,8,10$, and 16, with the same temporal pulse duration at full width half maximum (FWHM) $\tau=1$ ps, are shown in Fig 1 . We note that similar to pure-quartic solitons (PQSs), the temporal shapes of the PHEOD solitons exhibit oscillations in the tails [18,21], which become more prominent with increasing dispersion order, as seen in Fig. 1(b). This result can be understood using an analysis of the tails of the solutions $[18,21]$. Since the intensity is low there, we may neglect the nonlinear term in Eq. (3), and $A_{k}$ can then be written as a linear combination of $k$ exponential terms $\exp \left(\lambda_{k} T\right)$, where the $\lambda_{k}$ are the $k$ roots of

$$
\lambda_{k}^{k}=(-1)^{k / 2} \frac{k !}{\left|\beta_{k}\right|} \mu .
$$
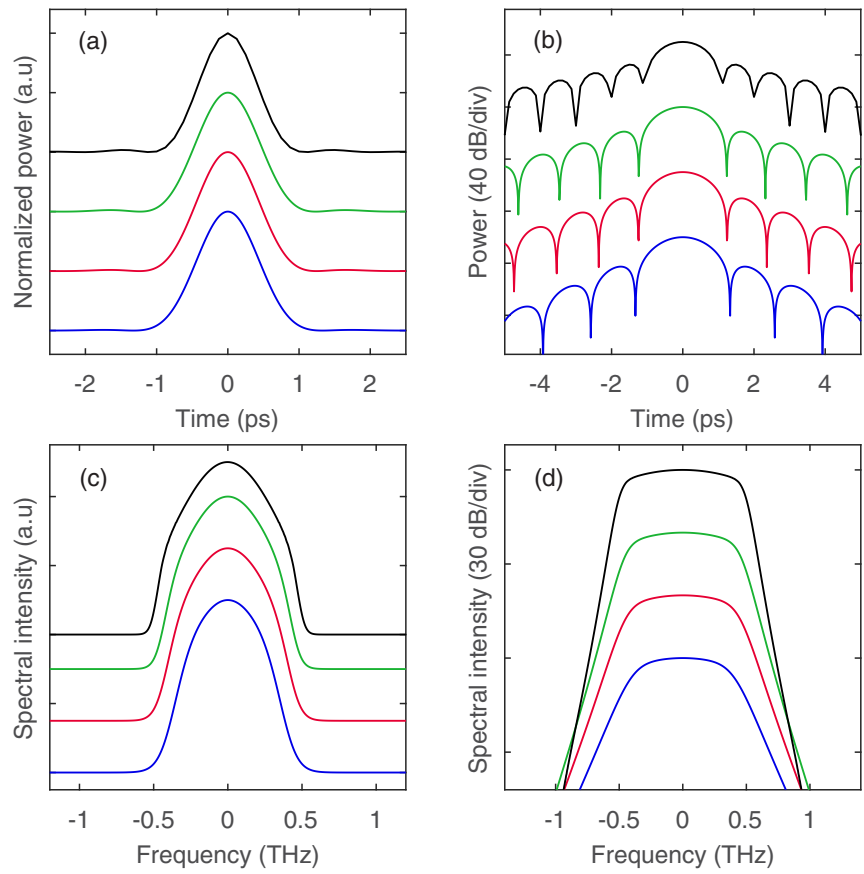

FIG. 1. Numerically calculated temporal stationary solution for $k=6$ (blue), 8 (red), 10 (green), and 16 (black) with a same pulse width (FWHM) of $\tau=1$ ps. Temporal profiles in linear (a) and logarithmic (b) scales. Corresponding linear (c) and logarithmic (d) spectra. The different solutions have been shifted vertically for clarity.

In the leading edge of a PHEOD soliton $(T<0)$ only the roots with positive real parts have nonzero coefficients, while conversely in the trailing edge only the roots with negative real parts are nonzero. One would expect that the tail is dominated by the exponential terms with the slowest decay rate. Consequently, the tails take the approximate form

$$
A_{k}(T) \propto e^{ \pm\left|\left(\frac{k ! \mu}{\beta_{k}}\right) \frac{1}{k}\right| \sin \left(\frac{\pi}{k}\right) T} \cos \left[\left|\left(\frac{k ! \mu}{\beta_{k}}\right)^{\frac{1}{k}}\right| \cos \left(\frac{\pi}{k}\right) T+\varphi\right],
$$

where $\varphi$ is determined by the nonlinear effects near the peak, and where the \pm signs refer to the leading and trailing edges, respectively. This result shows that, except for the conventional case $k=2$, PHEOD solitons have oscillatory tails with an exponentially decreasing envelope [18,21]. As $k$ increases, the oscillations become denser; according to Eq. (6), the envelope of the intensity changes by a factor $e^{-2 \pi \tan (\pi / k)}$ between consecutive nodes in the tails, consistent with Fig. 1(b).

As seen in Figs. 1(c) and 1(d), the central part of the PHEOD soliton spectra become increasingly flat as the dispersion order $k$ increases. To understand this, recall that the second derivative of a function corresponds to the second moment of its Fourier transform [32]. A set function with increasing oscillations has an increasingly lower second moment, and the associated Fourier transform is thus increasingly flat [18]. Physically, this can be understood from Eq. (1) for the group velocity near frequency $\omega_{0}$. For high dispersion orders $k$ the group velocity remains approximately constant around $\omega_{0}$ before changing rapidly. There is thus a frequency 
TABLE I. Key PHEOD soliton parameters versus order $k$. Column 2: flatness parameter $F$. Column 3: relation between peak power and nonlinear phase shift $\mu / \gamma P_{0}$, where $P_{0}$ is the peak power. Column 4: energy constant $M_{k}$ from Eq. (7). Column 5: $N_{k}$ from Eq. (8). All constants are dimensionless.

\begin{tabular}{|c|c|c|c|c|}
\hline \multicolumn{3}{|c|}{ Flatness } & \multirow{2}{*}{$\begin{array}{c}\text { Energy coefficient } \\
M_{k} \text { [Eq. (7)] }\end{array}$} & \multirow{2}{*}{$\begin{array}{c}\text { Peak power coefficient } \\
N_{k} \text { [Eq. (8)] }\end{array}$} \\
\hline$k$ & $F$ & $\mu /\left(\gamma P_{0}\right)$ & & \\
\hline 2 & 0.707 & 0.500 & 3.54 & 3.11 \\
\hline 4 & 0.854 & 0.620 & 2.87 & 2.71 \\
\hline 6 & 0.876 & 0.658 & 0.94 & 0.90 \\
\hline 8 & 0.899 & 0.674 & 0.162 & 0.156 \\
\hline 10 & 0.905 & 0.682 & $1.78 \times 10^{-2}$ & $1.64 \times 10^{-2}$ \\
\hline 12 & 0.912 & 0.687 & $1.16 \times 10^{-3}$ & $7.02 \times 10^{-4}$ \\
\hline 14 & 0.920 & 0.690 & $5.86 \times 10^{-5}$ & $5.78 \times 10^{-5}$ \\
\hline 16 & 0.925 & 0.693 & $2.50 \times 10^{-6}$ & $2.17 \times 10^{-6}$ \\
\hline
\end{tabular}

interval around $\omega_{0}$ for which the dispersion is essentially irrelevant, and in this frequency interval the spectral intensity does not need to vary significantly to balance the dispersion. We quantify flatness of the spectrum in the next section.

\section{B. General properties of PHEOD solitons}

As discussed above, the spectral flatness of the PHEOD solitons increases with the dispersion order $k$. The flatness or peakedness of a function is often expressed in terms of the kurtosis, but this measure has been discredited [33]. Instead we introduce the flatness $F$, which we define to be the fraction of the pulse energy that is within its spectral FWHM. Since it is a fraction, $F$ is intrinsically normalized: it has maximum $F=1$ for a rectangular function and $0<F<1$ for all other functions. The flatness $F$ is an intrinsic property of a function and does not depend on its parameters. For example, for all Gaussian functions $F=\operatorname{erf}(\sqrt{\ln 2}) \approx 0.761$, where $\operatorname{erf}(x)$ is the error function, and $F=1 / \sqrt{2} \approx 0.707$ for all squared hyperbolic secants. The values of $F$ are listed in the second column of Table I; they increase monotonically as shown in Fig. 1(d), quantifying the increased flatness of the spectrum.

From the numerical solutions we can extract several other PHEOD soliton properties. First, we evaluate the ratio between $\mu$ and $\gamma P_{0}$ for $k=2$ to $k=16$, where $P_{0}$ is the peak power. Numerical results for $k>16$ are not reliable due to difficulties evaluating high-order derivatives. We know that for $k=2, \mu=\gamma P_{0} / 2$ whereas $\mu=0.62 \gamma P_{0}$ for $k=4$ [18]. As shown in Column 3 of Table I, as $k$ increases, the ratio $\mu /\left(\gamma P_{0}\right)$ increases as well but seems to saturate at a value close to 0.7. One implication of this is that near the PHEOD soliton peak the three terms in Eq. (3) have the same order of magnitude. In particular this implies that the second and third terms in Eq. (3) have the same order of magnitude. We use this result below.

Following the scaling argument of Eq. (4) and by dimensional analysis, we find that the energy-width scaling relation of PHEOD solitons for the $k$ th order of dispersion takes the form

$$
E_{k}=\frac{M_{k}\left|\beta_{k}\right|}{\gamma \tau^{k-1}}
$$

where the energy coefficient $M_{k}$ is to be found numerically (except for $k=2$ ). The results are summarized in Column 4 of Table I. Similarly, we define the peak power coefficients $N_{k}$ as relating the peak power to the pulse length

$$
P_{0}=\frac{N_{k}\left|\beta_{k}\right|}{\gamma \tau^{k}}
$$

with the results shown in the final column of Table I. Note that $N_{k}$ can be thought of as relating the balance of the nonlinear and dispersive effects.

Table I shows that the only coefficients that vary substantially with $k$ are $M_{k}$ and $N_{k}$ : both are of order unity for small $k$ but decrease rapidly with increasing $k$ and are well below $10^{-5}$ for $k=16$. Their ratio is approximately constant, which, consistent with Fig. 1(a), reflects that the temporal pulse shape does not change substantially. This means that the PHEOD soliton energy and peak lower are lower than might be expected from the favorable energy scaling relation.

We now discuss the implications of the sharp decrease in $M_{k}$ and $N_{k}$ with $k$. We noted earlier that the fact that $\mu /\left(\gamma P_{0}\right)$ remains of order unity implies that near the PHEOD soliton peak the second and third terms in Eq. (3) have the same order of magnitude. We may therefore write

$$
\left|\frac{\beta_{k}}{k !} \frac{\partial^{k} A_{k}}{\partial T^{k}}\right| \sim\left|\gamma P_{0} A_{k}\right|
$$

where $\sim$ indicates that the two sides have the same order of magnitude. Using Eq. (8) we may then write

$$
\left|\frac{1}{k !} \frac{\partial^{k} A_{k}}{\partial T^{k}}\right| \sim\left|\frac{N_{k} A_{k}}{\tau^{k}}\right| \text {. }
$$

The derivative on the left-hand side is taken with respect to $T$; if we consider the derivative with respect to the dimensionless quantity $T / \tau$ then the equation reduces to

$$
\left|\frac{1}{k !} \frac{\partial^{k} A_{k}}{\partial(T / \tau)^{k}}\right| \sim\left|N_{k} A_{k}\right| .
$$

Using Eqs. (3) and (11) we may write $A_{k}(T / \tau)$ in a Taylor series

$$
A_{k}(T / \tau) \sim \sum_{p=0}^{\infty} \mathcal{A}_{k, p}(T / \tau)^{p}, \quad \mathcal{A}_{k, p}=\Lambda_{k, p} \frac{\left(k ! N_{k}\right)^{p / k}}{p !},
$$

where $\Lambda_{k, p}$ is a linear combination of $p$ powers of $k$ th roots of \pm 1 . The expression for $\mathcal{A}_{k, p}$ is consistent with Eq. (5) with $\mu \sim \gamma P_{0}$. Recall that the rate at which the coefficient of Taylor series decrease is related to the expansion's radius of convergence [34]: an infinite radius of convergence requires $\left|\mathcal{A}_{k, p+1} / \mathcal{A}_{k, p}\right| \rightarrow 0$ as $p \rightarrow \infty$. This is satisfied here since

$$
\frac{\mathcal{A}_{k, p+1}}{\mathcal{A}_{k, p}}=\frac{\Lambda_{k, p+1}}{\Lambda_{k, p}} \frac{\left(k ! N_{k}\right)^{1 / k}}{p+1},
$$

with $\Lambda_{k, p+1} / \Lambda_{k, p}$ finite as $\Lambda_{k, i}$ is a finite sum of $k$ terms and $\left|\Lambda_{k, i}\right|=\left|\Lambda_{k, i+k}\right|$. This result implies that the rapid decrease of $M_{k}$ and $N_{k}$ with $k$ is related to the infinite radius of convergence of its Taylor expansion. One implication of this result is that for high order of dispersion $k$, the decrease of the energy constant $M_{k}$ might hamper the advantageous energywidth scaling. Therefore, there may exist an optimal order of 


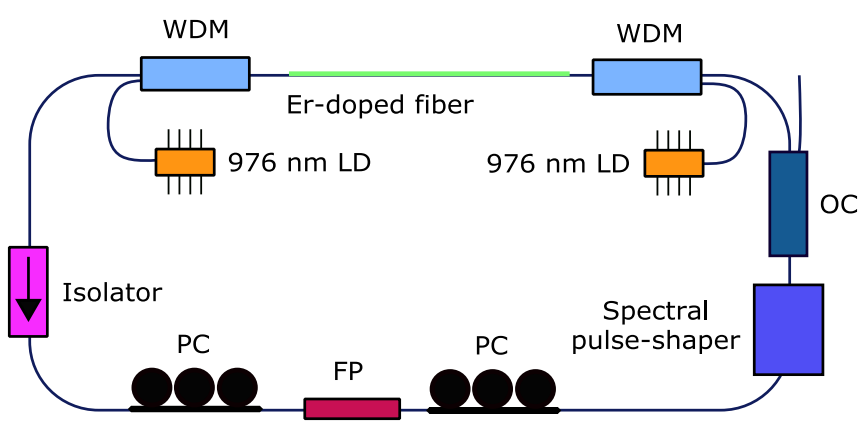

FIG. 2. Schematic of the erbium-doped laser cavity with the following components: Er, erbium; WDM, wavelength division multiplexer; LD, laser diode; OC, output coupler; PC, polarization controller; FP, fiber polarizer.

dispersion for the generation of ultrashort high-energy soliton pulses.

\section{EXPERIMENTAL RESULTS}

\section{A. Experimental setup}

The intrinsic dispersion of conventional optical wave guides is dominated by quadratic contribution $\left(\beta_{2}\right)$ while the effects of higher-order dispersion are usually weak. In fact, a complex structure was required just to achieve dominant negative quartic dispersion [17,35]. This strongly limits the possibility of observing the propagation of PHEOD solitons in waveguides. To overcome this limitation, and achieve the dominant negative high-order dispersion required for the generation of these solitons, we used a passively mode-locked fiber laser similar to the one reported in Ref. [19]. The laser configuration used in our experiments is shown schematically in Fig. 2. It incorporates an intracavity programmable spectral shaper (Finisar WaveShaper), which is used to adjust the net-cavity dispersion $[19,36,37]$. The applied phase mask compensates for the intrinsic second-, third-, and fourth-order dispersion of the fiber components and applies a large negative, high even-order dispersion. The applied phase profile can be written as

$$
\phi(\omega)=L\left[\sum_{n=2}^{4} \frac{\beta_{n}\left(\omega-\omega_{0}\right)^{n}}{n !}+\frac{\beta_{k}\left(\omega-\omega_{0}\right)^{k}}{k !}\right],
$$

where $L=18.17 \mathrm{~m}$ is the cavity length, and $\beta_{n}$ is the $n$th dispersion order for $n=2,3$, and 4 to account for the cavity dispersion. For the results presented in this work $\beta_{2}=+21.4 \mathrm{ps}^{2} / \mathrm{km}, \beta_{3}=-0.12 \mathrm{ps}^{3} / \mathrm{km}$, and $\beta_{4}=+2.2 \times$ $10^{-3} \mathrm{ps}^{4} / \mathrm{km}$. These values are chosen to compensate for the dispersion of the SMF used in our setup and are based on values reported in Refs. [38,39]. The second term on the right-hand side of Eq. (14) corresponds to the negative high, even-order dispersion required for the generation of sextic $(k=6)$, octic $(k=8)$, or decic $(k=10)$ PHEOD solitons. To obtain the complete spectral and temporal characterization of the pulses, we used a frequency-resolved electrical gating (FREG) setup which allows for the measurement of the pulse spectrogram [40]. The temporal intensity and phase of the pulses are then retrieved using a conventional blind deconvolution numerical algorithm [41].

\section{B. Spectral and temporal characterization}

The results of the spectral, temporal, and phase-resolved measurements of the output pulses for the laser operating with net pure-sextic, -octic, and -decic dispersion are shown in the first, second, and third rows of Fig. 3, respectively. Figures 3(a)-3(c) (left column) show the measured output spectra (blue curves) and the corresponding numerically calculated pulse shapes (red-dashed curves), for the three different dispersion orders. The measured spectral $-3 \mathrm{~dB}$ bandwidths are $\Delta \lambda=3.9,3.8$, and $3.8 \mathrm{~nm}$, for the sextic, octic, and decic PHEOD solitons, respectively. For all cases the experimental and numerically calculated spectra agree well. The spectral fluctuations away from the pulses arise from the limited spectral resolution of the pulse shaper. For rapidly varying functions, it undersamples the phase profile leading to aliasing in the applied phase mask, particularly for the highest dispersion order for which the phase varies rapidly for frequencies away from $\omega_{0}$. Since the spectral fluctuations thus appear far from the central frequency and at least $10 \mathrm{~dB}$ below the peak [see Fig. 3(c)], we are confident that they do not affect the pulse dynamics significantly.

This assertion is confirmed by the corresponding measured spectrograms in Figs. 3(d)-3(f), which show clear pulses for all three cases. The vertical streaks at short and long wavelengths correspond to the first sidebands [42]. Finally, the temporal intensity and phase profiles of the sextic, octic, and decic PHEOD solitons are shown in Figs. 3(g)-3(i), respectively. The retrieved FWHM pulse durations of the pure-sextic, -octic, and -decic solitons are $\tau=1.68,1.69$, and $1.77 \mathrm{ps}$, respectively. For all cases, the measured temporal intensities (blue curves) are in good agreement with the corresponding numerical solutions (red-dashed curves) for similar FWHM. The retrieved temporal phase (orange curve) indicates that the emitted pulses are slightly chirped. This is because our experimental setup is a lumped system in which the required dispersion is applied at a single point in the cavity, just before the output coupler [19]. Note that the numerically predicted oscillations in the tails of the temporal profiles [see Fig. 1(b)] are not observed since these are expected to appear approximately $20 \mathrm{~dB}$ below the pulse's maximum, which is below the background in our experiments.

For all cases we estimated the experimental flatness parameter $F$, defined in the previous section. The red circles in Fig. 4 give the flatness $F$ of the numerically calculated spectra from Fig. 1 for different even dispersion order $k$. The measured values of $F$ (blue diamonds) from the spectra from Fig. 3 and Ref. [19] agree well with the numerically calculated ones and confirm that the flatness of the spectrum increases monotonically with $k$. It has been shown that flatter spectra can lead to enhanced pump-comb conversion and smaller line-to-line power variations in frequency combs [30].

\section{Sideband analysis}

To confirm the nature of the cavity's linear dispersion, we analyzed the position of the spectral sidebands of the emitted pulses. These dispersive waves arise from the constructive interference between the solitons and the linear waves emitted by the soliton while it propagates inside the cavity $[19,42]$. Constructive interference occurs when 

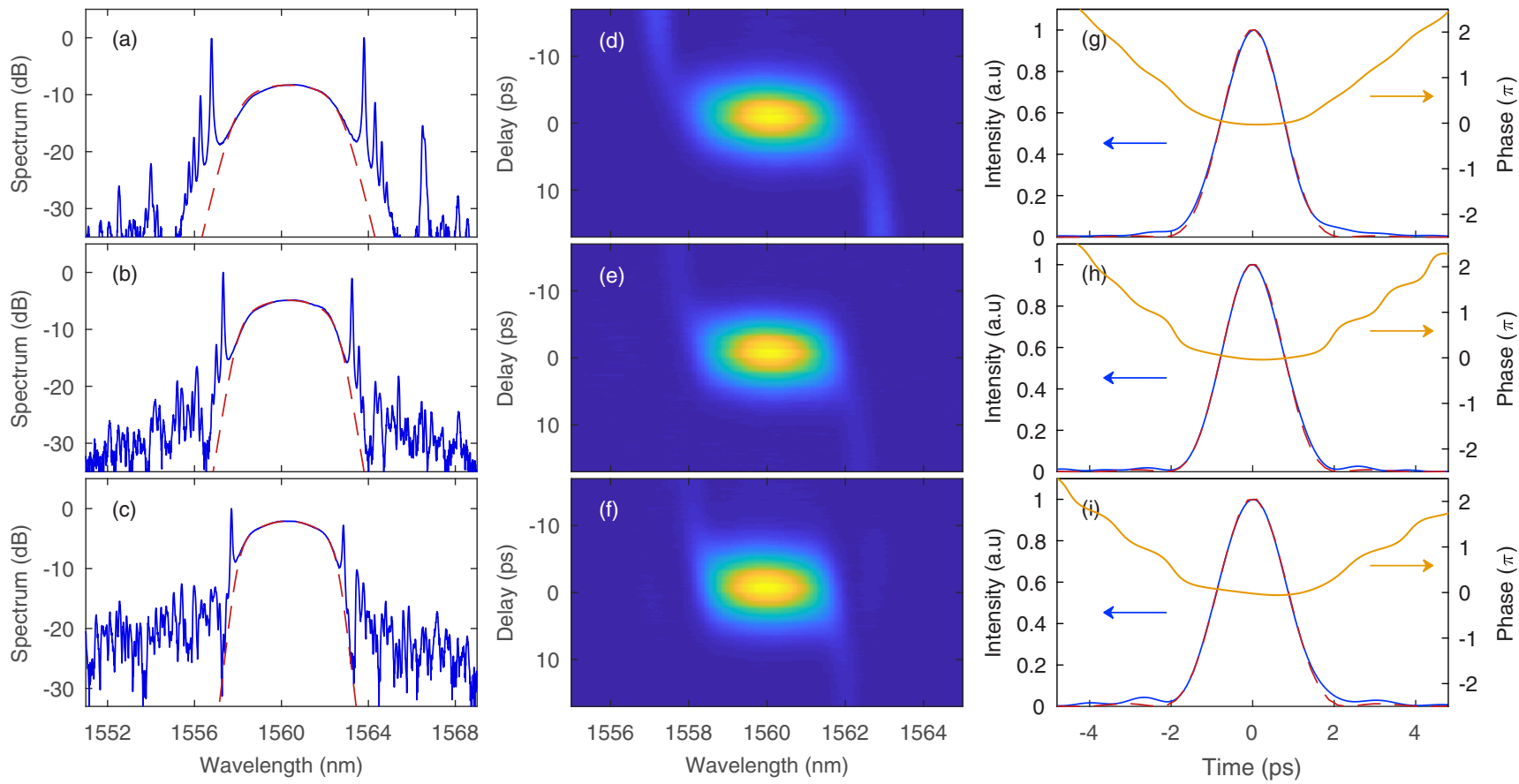

FIG. 3. Spectral and temporal measurements of pure high-order dispersion solitons: sextic (top row), octic (middle row), and decic (bottom row) dispersion. The applied dispersion is $\beta_{6}=-500 \mathrm{ps}^{6} / \mathrm{km}, \beta_{8}=-15 \times 10^{3} \mathrm{ps}^{8} / \mathrm{km}$, and $\beta_{10}=-1 \times 10^{6} \mathrm{ps}^{10} / \mathrm{km}$, respectively. (a)(c) Measured (blue) and calculated (red-dashed) spectra. (d)-(f) Measured spectrograms. (g)-(i) Retrieved temporal intensity (blue), phase (orange), and corresponding calculated temporal shapes (red-dashed).

$\beta_{\text {sol }}-\beta_{\text {lin }}=2 m \pi / L$ where $m$ is a positive integer. For $k$ thorder dispersion the linear waves satisfy $\beta_{\text {lin }}=-\left|\beta_{k}\right|(\omega-$ $\left.\omega_{0}\right)^{k} / k$ !, while the PHEOD solitons have a constant dispersion across their entire bandwidth of $\beta_{\text {sol }}=C_{k}\left|\beta_{k}\right| / \tau^{k}[18,43]$, where $C_{k}$ are constants of order unity that depend on the dispersion order. Thus, we find that the spectral position of the $m$ th spectral sideband $\omega_{m}$ is given by

$$
\omega_{m}= \pm \frac{1}{\tau}\left[k !\left(\frac{m \pi \tau^{k}}{\left|\beta_{k}\right| L}-C_{k}\right)\right]^{1 / k} .
$$

Following this argument, it is straightforward to show that for a pure $k$ th-order dispersion soliton, the $k$ th power of two consecutive sidebands is constant and given by $2 \pi k ! /\left(\left|\beta_{k}\right| L\right)$, irrespective of the value of $C_{k}$. To check this prediction, for each dispersion order we measured the output spectrum for three different values of the dispersion coefficient $\beta_{k}$, and we

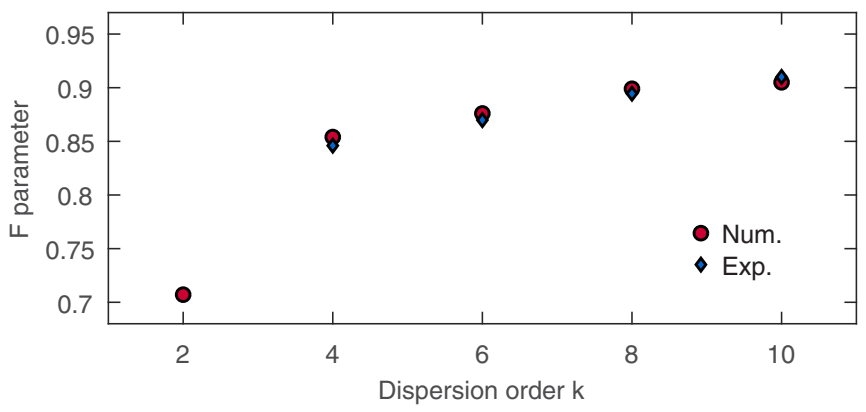

FIG. 4. Numerical (red circles) and experimental (blue diamonds) values of the flatness $F$ of the PHEOD soliton spectrum versus dispersion order. measured the spectral positions of the low-frequency sidebands.

The results of these measurements are shown in Fig. 5. In Figs. 5(a)-5(c), we show three measured spectra for each dispersion order $k$. The sextic PHEOD soliton spectra for three different values of $\beta_{6}$ are shown in Fig. 5(a). Corresponding results for octic and decic PHEOD solitons are shown in Figs. 5(b) and 5(c), respectively. The circles mark the spectral positions of the low-frequency sidebands. The $k$ th power of these measured positions as a function of the sideband order for the nine PHEOD soliton spectra are shown in Figs. 5(d)5(f). In all cases the spacings follow a linear relationship as expected. The predicted and measured spectral spacing for all the spectra shown in Figs. 5(a)-5(c) are summarized in Table II. Note that the experimental values agree within $4 \%$ to the corresponding expected values calculated from Eq. (15) and based on the net-cavity dispersion that was applied by the pulse shaper. Results for the high-frequency sidebands (not shown here) are similarly close to the expected values. Since taking a high power of a data set amplifies the noise, the agreement between the measured and expected results is remarkable, confirming the type and magnitude of the cavity dispersion.

\section{Energy-width scaling}

Finally, we study the energy-width scaling relationship of the PHEOD solitons. As discussed in Sec. II B and following the scaling argument, the energy-width scaling relation of PHEOD solitons is proportional to $\tau^{-(k-1)}$. We experimentally measured the output pulse energy as a function of the pulse duration for three different values of dispersion $\beta_{k}$, for 

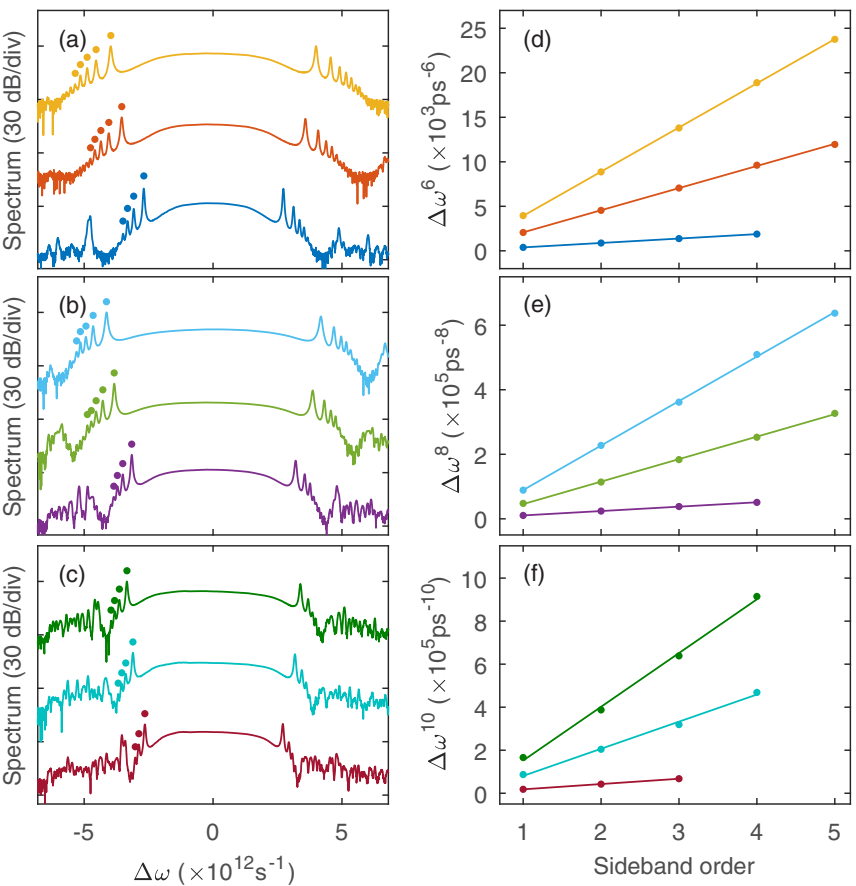

FIG. 5. Measured PHEOD soliton output spectra for different dispersion orders. (a) Sextic PHEOD soliton spectra for $\beta_{6}=$ -50 (yellow), $\beta_{6}=-100$ (orange), and $\beta_{6}=-500 \mathrm{ps}^{6} / \mathrm{km}$ (blue). (b) Octic PHEOD soliton spectra for $\beta_{8}=-100$ (cyan), $\beta_{8}=-200$ (green), and $\beta_{8}=-1000 \mathrm{ps}^{8} / \mathrm{km}$ (purple). (c) Decic PHEOD soliton spectra for $\beta_{10}=-5000$ (green), $\beta_{10}=-10 \times 10^{3}$ (cyan), and $\beta_{10}=-50 \times 10^{3} \mathrm{ps}^{10} / \mathrm{km}$ (red). Colored circles show the $k$ th power of the measured sidebands positions versus sideband order for the (d) sextic, (e) octic, and (f) decic PHEOD soliton spectra. The solid lines correspond to linear fits.

each order of dispersion considered. Concretely, we adjusted the pump power in the laser cavity and measured the output pulse energy after deducting the portion of energy in the spectral sidebands by integrating the optical spectrum. The results of these measurements for $k=6,8$, and 10 are shown in Fig. 6. The circles show the measured pulse energies versus the pulse duration $(\tau)$ for three different values of dispersion $\beta_{k}$ for each dispersion order $k$. All results are in good agreement with Eq. (7) once we account for the output coupling and

TABLE II. Predicted [from Eq. (15)] and measured sideband spacing values for different values of applied dispersion $k$.

\begin{tabular}{lccc}
\hline \hline $\begin{array}{l}\text { Dispersion } \\
\text { order } k\end{array}$ & $\begin{array}{c}\text { Applied } \beta_{k} \\
\left(\mathrm{ps}^{k} / \mathrm{km}\right)\end{array}$ & $\begin{array}{c}\text { Predicted } \\
\text { spacing }\left(\mathrm{ps}^{-k}\right)\end{array}$ & $\begin{array}{c}\text { Measured } \\
\text { spacing }\left(\mathrm{ps}^{-k}\right)\end{array}$ \\
\hline \multirow{3}{*}{6} & -50 & $4.98 \times 10^{3}$ & $4.96 \times 10^{3}$ \\
& -100 & $2.49 \times 10^{3}$ & $2.48 \times 10^{3}$ \\
& -500 & $4.98 \times 10^{2}$ & $4.97 \times 10^{2}$ \\
8 & -100 & $1.39 \times 10^{5}$ & $1.38 \times 10^{5}$ \\
& -200 & $6.97 \times 10^{4}$ & $6.96 \times 10^{4}$ \\
& -1000 & $1.39 \times 10^{4}$ & $1.36 \times 10^{4}$ \\
10 & -5000 & $2.51 \times 10^{5}$ & $2.49 \times 10^{5}$ \\
& $-10 \times 10^{3}$ & $1.25 \times 10^{5}$ & $1.26 \times 10^{5}$ \\
& $-50 \times 10^{3}$ & $2.51 \times 10^{4}$ & $2.46 \times 10^{4}$ \\
\hline \hline
\end{tabular}

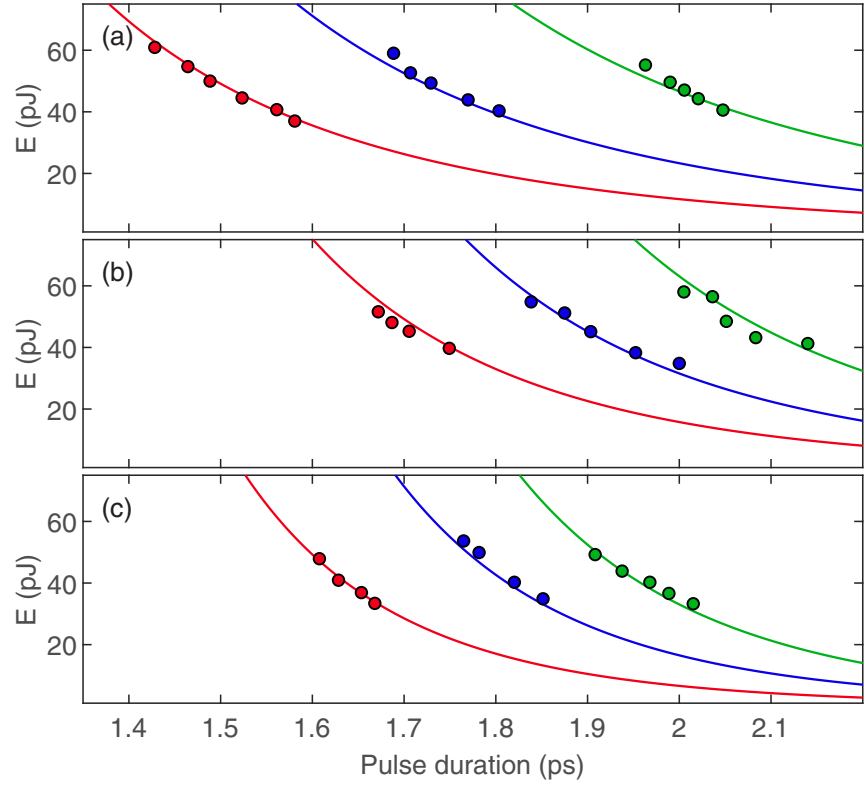

FIG. 6. Measurement of energy-width scaling properties of the pure high-order dispersion Kerr solitons. The circles mark the measured pulse energy $E$ versus pulse duration. (a) Puresextic soliton energy for $\beta_{6}=-500$ (red), $\beta_{6}=-1000$ (blue), and $\beta_{6}=-2000 \mathrm{ps}^{6} / \mathrm{km}$ (green). (b) Pure-octic soliton energy for $\beta_{8}=-15 \times 10^{3}$ (red), $\beta_{8}=-30 \times 10^{3}$ (blue), and $\beta_{8}=-60 \times$ $10^{3} \mathrm{ps}^{8} / \mathrm{km}$ (green). (c) Pure-decic soliton energy for $\beta_{10}=-2 \times$ $10^{5}$ (red), $\beta_{10}=-5 \times 10^{5}$ (blue), and $\beta_{10}=-1 \times 10^{6} \mathrm{ps}^{10} / \mathrm{km}$ (green). The solid curves are fits for (a) pure-sextic, (b) -octic, and (c) -decic solitons from Eq. (7).

the insertion loss of the pulse shaper. This confirms that these pure high-order dispersion solitons follow a different energywidth scaling relation that could be used for the generation of ultrashort optical pulses with high energy.

\section{CONCLUSION AND DISCUSSION}

We report the experimental discovery of an entire family of optical solitons arising from the balance between SPM and higher-order dispersion. One can consider conventional optical solitons to be the lowest-order member of this family of PHEOD solitons. All these pulses fundamentally arise from similar physical effects: the nonlinearity generates frequencies on the pulses' leading and trailing edges, and these shift towards the pulse center under the effect of dispersion. Our investigation combines numerical results following from solving Eq. (3) and experimental results obtained using a fiber laser. The fiber laser incorporates a spectral pulse shaper, which is used to apply a large negative pure high, even-order dispersion [19]. We find that the numerical and experimental results are in very good agreement.

While our experimental approach differs from the conservative system described by Eq. (2), it allows for an unprecedented level of dispersion control, which is currently impossible through conventional waveguide dispersion engineering [17]. While we demonstrate PHEOD solitons only up to the 10th order of dispersion, we emphasize that our approach is limited only by the specifications of the pulse 
shaper. This limitation could be overcome by using a device with higher spectral resolution and bandwidth so enabling the generation of PHEOD solitons of order higher than 10th. We have shown that our setup provides a powerful tool to open up new routes for the generation and study of a wide range of optical pulses [44].

One of the insights afforded by our analysis is a study of the PHEOD solitons as their order $k$ increases. A key advantage of PQS over conventional solitons is the scaling relation $E_{4} \propto \tau^{-3}$ [19]. Even though the scaling becomes increasingly advantageous as $k$ increases, as illustrated by Eq. (7), the fact that the coefficient $M_{k}$ decreases so rapidly leads to pause for thought. While it is always possible to increase the energy by increasing the magnitude of the dispersion coefficient $\beta_{k}$, it may imply the existence of an optimal, finite value of $k$ for maximizing pulse energy. We note that an additional advan- tage of using high-order PHEOD solitons is that their spectra become increasingly flat. This could be used to generate frequency combs with small tooth power variations $[30,45]$.

In addition to direct quantitative insights on soliton pulses provided by our experiments, the approach itself is expected to become an established tool for the generation and study of ultrafast pulses $[19,44]$. We expect our results to stimulate future investigations and discoveries in other areas of physics, engineering, and applied mathematics.

\section{ACKNOWLEDGMENT}

We acknowledge funding from the Australian Research Project (ARC) Discovery Project (DP180102234) and the Asian Office of Aerospace R\&D (AOARD) grant (FA238619-1-4067).
[1] E. Polturak, P. G. N. deVegvar, E. K. Zeise, and D. M. Lee, Solitonlike Propagation of Zero Sound in Superfluid ${ }^{3} \mathrm{He}$, Phys. Rev. Lett. 46, 1588 (1981).

[2] J. Denschlag, J. E. Simsarian, D. L. Feder, C. W. Clark, L. A. Collins, J. Cubizolles, L. Deng, E. W. Hagley, K. Helmerson, W. P. Reinhardt et al., Generating solitons by phase engineering of a Bose-Einstein condensate, Science 287, 97 (2000).

[3] L. F. Mollenauer, M. J. Neubelt, M. Haner, E. Lichtman, S. G. Evangelides, and B. M. Nyman, Demonstration of error-free soliton transmission at $2.5 \mathrm{Gbit} / \mathrm{s}$ over more than $14,000 \mathrm{~km}$, Electronic Lett. 27, 2055 (1991).

[4] H. A. Haus and W. S. Wong, Solitons in optical communications, Rev. Mod. Phys. 68, 423 (1996).

[5] T. Herr, V. Brasch, J. D. Jost, C. Y. Wang, N. M. Kondratiev, M. L. Gorodetsky, and T. J. Kippenberg, Temporal solitons in optical microresonators, Nat. Photonics 8, 145 (2014).

[6] X. Yi, Q. F. Yang, K. Y. Yang, M. G. Suh, and K. Vahala, Soliton frequency comb at microwave rates in a high- $Q$ silica microresonator, Optica 2, 1078 (2015).

[7] L. F. Mollenauer and R. H. Stolen, The soliton laser, Opt. Lett. 9, 13 (1984)

[8] J. Zhou, G. Taft, C. P. Huang, M. M. Murnane, H. C. Kapteyn, and I. P. Christov, Pulse evolution in a broad-bandwidth Ti:sapphire laser, Opt. Lett. 19, 1149 (1994).

[9] J. N. Elgin, Soliton propagation in an optical fiber with thirdorder dispersion, Opt. Lett. 17, 1409 (1992).

[10] Y. Kodama, M. Romagnoli, S. Wabnitz, and M. Midrio, Role of third-order dispersion on soliton instabilities and interactions in optical fibers, Opt. Lett. 19, 165 (1994).

[11] M. L. Dennis and I. N. Duling, Third-order dispersion in femtosecond fiber lasers, Opt. Lett. 19, 1750 (1994).

[12] M. Santagiustina, Third-order dispersion radiation in solid-state solitary lasers, J. Opt. Soc. Am. B 14, 1484 (1997).

[13] A. Höök and M. Karlsson, Ultrashort solitons at the minimumdispersion wavelength: Effects of fourth-order dispersion, Opt. Lett. 18, 1388 (1993).

[14] K. C. Chan and H. F. Liu, Effect of third-order dispersion on soliton-effect pulse compression, Opt. Lett. 19, 49 (1994).
[15] A. B. Aceves, C. D. Angelis, G. Nalesso, and M. Santagiustina, Higher-order effects in bandwidth-limited soliton propagation in optical fibers, Opt. Lett. 19, 2104 (1994).

[16] I. P. Christov, M. M. Murnane, H. C. Kapteyn, J. Zhou, and C.-P. Huang, Fourth-order dispersion-limited solitary pulses, Opt. Lett. 19, 1465 (1994).

[17] A. Blanco-Redondo, C. M. de Sterke, J. E. Sipe, T. F. Krauss, B. J. Eggleton, and C. Husko, Pure-quartic solitons, Nature Comm. 7, 10427 (2016).

[18] K. K. K. Tam, T. J. Alexander, A. Blanco-Redondo, and C. M. de Sterke, Stationary and dynamical properties of pure-quartic solitons, Opt. Lett. 44, 3306 (2019).

[19] A. F. J. Runge, D. D. Hudson, K. K. K. Tam, C. M. de Sterke, and A. Blanco-Redondo, The pure-quartic soliton laser, Nat. Photonics 14, 492 (2020).

[20] M. Karlsson and A. Höök, Soliton-like pulses governed by fourth-order dispersion in optical fibers, Opt. Commun. 104, 303 (1994).

[21] N. Akhmediev, A. Buryak, and M. Karlsson, Radiationless optical solitons with oscillating tails, Opt. Commun. 110, 540 (1994).

[22] M. Piché, J.-F. Cormier, and X. Zhu, Bright optical soliton in the presence of fourth-order dispersion, Opt. Lett. 21, 845 (1996).

[23] V. E. Zhakarov and E. A. Kuznetsov, Optical solitons and quasisolitons, J. Exp. Theor. Phys. 86, 1035 (1998).

[24] S. Roy and F. Biancalana, Formation of quartic solitons and a localized continuum in silicon-based waveguides, Phys. Rev. A 87, 025801 (2013).

[25] D. J. Kedziora, A. Ankiewicz, A. Chowdury, and N. Akhmediev, Integrable equations of the infinite nonlinear Schrödinger equation hierarchy with time variable coefficients, Chaos 25, 103114 (2015).

[26] A. Ankiewicz, D. J. Kedziora, A. Chowdury, U. Bandelow, and $\mathrm{N}$. Akhmediev, Integrable equations of the infinite nonlinear Schrödinger equation hierarchy with time variable coefficients, Phys. Rev. E 93, 012206 (2016).

[27] W.-R. Sun, Breather-to-soliton transitions and nonlinear wave interactions for the nonlinear Schrödinger equation with the sextic operators in optical fibers, Ann. Phys. 529, 1600227 (2017). 
[28] S.-L. Jia, Y.-T. Gao, and L. Hu, Rogue waves, breather-tosoliton transitions and modulational instability for the nonlinear Schrödinger equation with octic operator in an optical fiber, Optik 142, 90 (2017).

[29] C. Bao, H. Taheri, L. Zhang, A. Matsko, Y. Yan, P. Liao, L. Maleki, and A. E. Willner, High-order dispersion in Kerr comb oscillators, J. Opt. Soc. Am. B 34, 715 (2017).

[30] H. Taheri and A. B. Matsko, Quartic dissipative solitons in optical Kerr cavities, Opt. Lett. 44, 3086 (2019).

[31] J. Yang, Newton-conjugate-gradient methods for solitary wave computations, J. Comput. Phys. 228, 7007 (2009).

[32] R. N. Bracewell, Fourier Transform and Its Applications (McGraw-Hill, New York, 1999).

[33] P. Westfall, Kurtosis as peakedness, 1905-2014. R.I.P., Am. Stat. 68, 191 (2014).

[34] H. Wyld, Mathematical Methods for Physics (Benjamin Cummings, Boulder, Colorado, 1976), Chap. 10.

[35] C. W. Lo, A. Stefani, C. M. de Sterke, and A. Blanco-Redondo, Analysis and design of fibers for pure-quartic solitons, Opt. Express 26, 7786 (2018).

[36] J. Schröder, S. Coen, T. Sylvestre, and B. J. Eggleton, Dark and bright pulse passive mode-locked laser with in-cavity pulseshaper, Opt. Express 18, 22715 (2010).

[37] J. Peng and S. Boscolo, Filter-based dispersion-managed versatile ultrafast fibre laser, Sci. Rep. 6, 25995 (2016).

[38] K. Hammani, B. Kibler, C. Finot, P. Morin, J. Fatome, J. M. Dudley, and G. Millot, Peregrine soliton generation and breakup in standard telecommunications fiber, Opt. Lett. 36, 112 (2011)

[39] T. Ito, O. Slezak, M. Yoshita, H. Akiyama, and Y. Kobayashi, High-precision group-delay dispersion measurements of optical fibers via fingerprint-spectral wavelength-to-time mapping, Photon. Res. 4, 13 (2016).

[40] C. Dorrer and I. Kang, Simultaneous temporal characterization of telecommunication optical pulses and modulators by use of spectrograms, Opt. Lett. 27, 1315 (2002).

[41] R. Trebino, K. W. DeLong, D. N. Fittinghoff, J. N. Sweetser, M. A. Krumbügel, and B. A. Richman, Measuring ultrashort laser pulses in the time-frequency domain using frequencyresolved optical gating, Rev. Sci. Instrum. 68, 3277 (1997).

[42] S. M. J. Kelly, Characteristic sideband instability of periodically amplified average soliton, Electronic Lett. 28, 806 (1992).

[43] N. Akhmediev and M. Karlsson, Cherenkov radiation emitted by solitons in optical fibers, Phys. Rev. A 51, 2602 (1995).

[44] J. P. Lourdesamy, A. F. J. Runge, T. J. Alexander, D. D. Hudson, A. Blanco-Redondo, and C. M. de Sterke, Two-color soliton molecules from a fiber laser in proceedings of the 2020 Conference on Lasers and Electro-Optics Pacific Rim (CLEO-PR), paper C2B2, https://www.osapublishing.org/abstract.cfm?uri= CLEOPR-2020-C2B_2.

[45] S.-W. Huang, H. Zhou, J. Yang, J. F. McMillan, A. Matsko, M. Yu, D.-L. Kwong, L. Maleki, and C. W. Wong, Mode-Locked Ultrashort Pulse Generation from On-Chip Normal Dispersion Microresonators, Phys. Rev. Lett. 114, 053901 (2015). 